\title{
Relationship between indeterminate or positive lateral margin and local recurrence after endoscopic resection of colorectal polyps
}

Authors

Institutions
Makomo Makazu', Taku Sakamoto ${ }^{1}$, Eriko So ${ }^{1}$, Yosuke Otake ${ }^{1}$, Takeshi Nakajima ${ }^{1}$, Takahisa Matsuda', Ryoji Kushima² Yutaka Saito ${ }^{1}$

${ }^{1}$ Endoscopy Division, National Cancer Center Hospital, Tokyo, Japan

2 Pathology Division, National Cancer Center Hospital, Tokyo, Japan submitted 9. June 2014 accepted after revision 13. February 2015

\section{Bibliography}

DOI http://dx.doi.org/

10.1055/s-0034-1391853

Published online: 5.5.2015

Endosc Int Open 2015; 03: E252-E257

(c) Georg Thieme Verlag KG Stuttgart · New York

E-ISSN 2196-9736

\section{Corresponding author}

Taku Sakamoto, MD

Endoscopy Division

National Cancer Center Hospital

5-1-1 Tsukiji, Chuo-ku

Tokyo 104-0045

Japan

Fax: +81-3-3545-3567

tasakamo@ncc.go.jp

\section{License terms}

(1) $\ominus \circledast$
Background and study aims: Although endoscopic resection is widely used for the treatment of superficial colorectal neoplasms, the rate of local recurrence of lesions with a positive or indeterminate lateral margin on histologic evaluation is unclear. We aimed to demonstrate the relationship between lateral margin status and local recurrence after the endoscopic resection of intramucosal colorectal neoplasms.

Patients and methods: We retrospectively collected the clinical and pathologic data for 844 endoscopically resected colorectal intramucosal neoplasms with a size of $10 \mathrm{~mm}$ or larger. We investigated the relationship between the local recurrence rate and the lateral margin status (categorized as LM0 [negative], LM1 [positive], or LMX [indeterminate]).

Results: In total, 389 lesions were evaluated as LM0 and showed no local recurrence. Of the 455

\section{Introduction}

Techniques of endoscopic resection, including polypectomy [1], and endoscopic mucosal resection (EMR) [2 -5], are widely used for treating superficial colorectal neoplasms. Endoscopic submucosal dissection (ESD), which has recently been developed $[6,7]$, was first applied for the treatment of early gastric cancer and then for the treatment of other cancers of the digestive tract, including colon and rectal cancers [8-10].

Endoscopically resected tumors are evaluated pathologically. As a method of evaluation, the resection margins are divided into two categories lateral margin and vertical margin [11]. When a tumor is determined to have a positive lateral margin, additional surgery is not immediately considered; instead, colonoscopic surveillance is performed if no other noncurative factors, such as a positive vertical margin, lymphovascular invasion, or a histologic type of poorly differentiated adenocarcinoma, is present [12]. However, lesions evaluated as LMX or LM1, 30 showed local recurrence within a median period of 6.3 months (range, 1.7-48.1) from the initial endoscopic resection. The local recurrence rate of the en blocLMX group (2.2\%) was significantly lower than that of the piecemeal-LMX group (15.2\%). Of the 30 cases of recurrence, 28 were successfully treated with a second endoscopic resection. Of the two lesions that showed further recurrence, one was treated with a third endoscopic resection, whereas the other - which was a piecemeal-LMX lesion - was eventually diagnosed as invasive cancer and treated with surgery.

Conclusions: The local recurrence rate was lower in the en bloc-LMX group than in the piecemealLMX group. Thus, we believe that en bloc-LMX lesions that are completely and confidently resected endoscopically can be treated as en bloc-LM0 lesions.

only a few reports have focused on the relationship between the lateral margin status and local recurrence [13].

In the present study, we aimed to determine the relationship between the lateral margin status, particularly a positive or indeterminate lateral margin, and local recurrence following the endoscopic resection of colorectal polyps.

\section{Patients and methods \\ $\nabla$}

\section{Patients}

We retrospectively collected clinical and pathologic data for endoscopically resected colorectal intramucosal neoplasms with a size of $10 \mathrm{~mm}$ or larger. A flowchart indicating the enrolment of cases in the present study appears in Fig. 1. In total, 1412 lesions that met the following criteria were included in our colonoscopy database: (1) colorectal polyps that were pathologically proven to be low grade or high grade dysplastic adeno- 


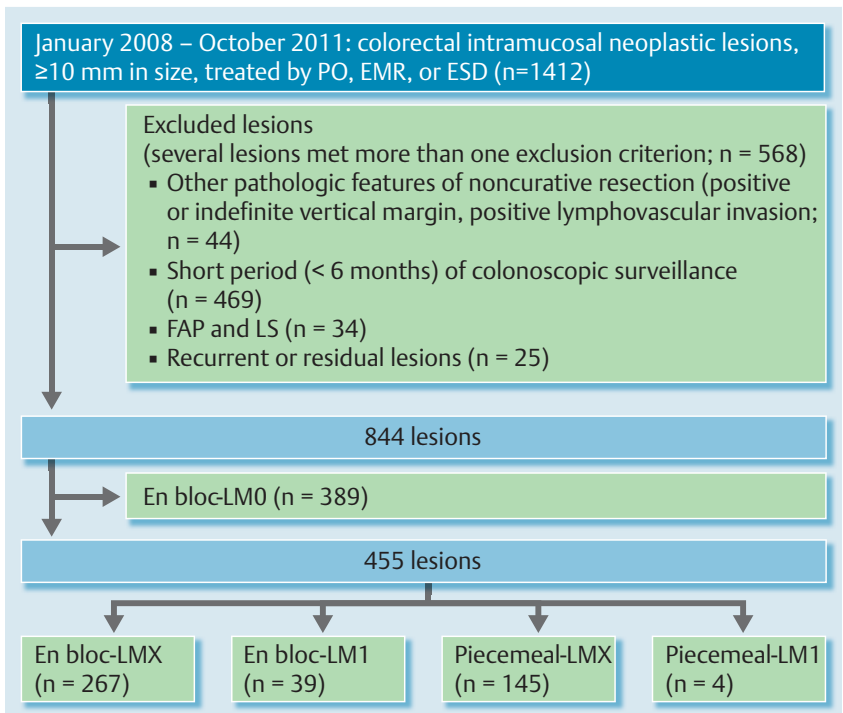

Fig. 1 Flowchart illustrating the inclusion criteria for the lesions examined in the present study of the relationship between an indeterminate or positive lateral margin and local recurrence after the endoscopic resection of colorectal polyps. PO, polypectomy; EMR, endoscopic mucosal resection; ESD, endoscopic submucosal dissection; FAP, familial adenomatous polyposis; LS, Lynch syndrome; LM0, lateral margin free of tumor cells; LMX, glands at the lateral margin could not be evaluated; LM1, lateral margin positive for tumor cells.

mas, (2) lesions with an endoscopically determined size of 10 $\mathrm{mm}$ or larger, and (3) lesions treated by polypectomy, EMR, or ESD at our institution between January 2008 and October 2011. Of these lesions, the lesions that met the following criteria were excluded: (1) lesions having pathologic features of noncurative resection (positive or indeterminate vertical margin or findings indicating lymphovascular invasion), (2) lesions with a short ( $<6$ months) period of surveillance following the initial endoscopic resection, (3) lesions associated with familial adenomatous polyposis or Lynch syndrome, and (4) recurrent or residual lesions.

Of the 844 remaining lesions, 389 lesions were pathologically evaluated as having a negative lateral margin. The other 455 lesions were evaluated as having an indeterminate or positive lateral margin. We retrospectively reviewed the endoscopic examination reports, pathologic reports, and medical charts of the patients with these lesions and extracted information on the location, macroscopic type, size, type of endoscopic treatment, pathologic findings of the lesions, and the presence or absence of local recurrence. In the present study, we used the Paris classification to describe the macroscopic types of the lesions [14]. Furthermore, in the present study, we defined en bloc resection as theresection of an entire lesion in one piece, as determined endoscopically. A casein which additional procedures were required for removal of the entire lesion after a one-piece resection was categorized as a piecemeal resection. All lesions that underwent piecemeal resection were categorized as having indeterminate or positive lateral margins.

Thus, 455 lesions were divided into four groups according to lateral margin status and type of endoscopic resection as follows: (1) en bloc-LMX group, lesions that underwent en bloc resection and showed an indeterminate lateral margin; (2) en bloc-LM1 group, lesions that underwent en bloc resection and showed a positive lateral margin; (3) piecemeal-LMX group, lesions that underwent piecemeal resection and showed an indeterminate lateral margin; and (4) piecemeal-LM1 group, lesions that underwent piecemeal resection and showed a positive lateral margin. The present study was approved by our institutional review committee, and informed consent was obtained from all patients for the colonoscopy and endoscopic resection. Furthermore, in our institution, we routinely receive informed consent for the use of our patients' specimens for our studies.

\section{Endoscopic procedure and surveillance}

For colonic preparation, the patients received 2 to $3 \mathrm{~L}$ of polyethylene glycol electrolyte solution on the day of the procedure. Thereafter, 10 to $20 \mathrm{mg}$ of scopolamine butylbromide was intravenously administered to all patients just before the procedure. The use of sedatives, such as midazolam, was left to the endoscopist's discretion.

Before endoscopic resection, the endoscopist observed the lesion through a magnifying colonoscope and determined the pit pattern according to the criteria established by Kudo et al., Fujii et al., and Matsuda et al. [15-17]. The pit patterns and borders of the lesions were accentuated by spraying the lesions with indigo carmine dye $(0.4 \%)$ and, if necessary, with $0.05 \%$ crystal violet. The capillary patterns of the lesions were classified with the use of narrow-band imaging [18].

In this study, we tended to choose ESD for lesions that met the following criteria [19]: (1) laterally spreading tumors (nongranular type) with a size of $20 \mathrm{~mm}$ or larger and (2) laterally spreading tumors (granular type) with a size of $40 \mathrm{~mm}$ or larger, both of which have a higher submucosal invasion rate and are difficult to treat with EMR or polypectomy; (3) lesions that could not be elevated with submucosal injection. However, these criteria were not strictly adhered to in all cases, and the endoscopist determined the appropriate procedure after considering several factors, such as the location of the lesion and the patient's general condition.

Magnifying video endoscopes (PCF-Q240Z, CF-H260AZI; Olympus, Tokyo, Japan) were used primarily for polypectomy and EMR. For ESD, the above-mentioned magnifying scopes were used only for observation, and other types of video endoscopes (GIF-Q260J, PCF-260JI; Olympus) were used for resection. Polypectomy and EMR were performed according to the well-recognized general method involving the use of a Snare Master (10, $25 \mathrm{~mm}$; Olympus). Snared polyps were first injected in patients undergoing EMR, but not in patients undergoing polypectomy. For ESD, we used a bipolar needle knife (B-Knife; Zeon Medical, Tokyo, Japan) and an insulation-tipped knife (ITknife; Olympus). In ESD procedures, the endoscopist injected glycerol and sodium hyaluronate (diluted in saline at a concentration of $1: 10$ ) into the submucosal layer under the tumor. Following endoscopic resection, we assessed for the presence of residual tumor by using a magnifying scope after spraying the site with indigo carmine dye. When residual neoplastic tissue was present at the edge of the resection site, additional snaring or hot biopsy was performed for removal.

The first surveillance colonoscopy evaluation was performed 3 to 6 months after the initial endoscopic resection.

\section{Pathologic evaluation}

All resected specimens were fixed in 10\% formalin for 24 hours. After fixation, pathologists cut the specimens in the following manner. In cases of ESD, the specimens were cut into slices 2 to $3 \mathrm{~mm}$ wide. In cases of polypectomy or EMR, if the size of the lesion was appropriate for sectioning into several specimens, the 
Table 1 Clinical characteristics of patients and lesions in a study of the relationship between indeterminate or positive lateral margin status and local recurrence after endoscopic resection of colorectal polyps.

\begin{tabular}{|c|c|c|c|c|c|}
\hline Characteristics of patients and lesions & $\begin{array}{l}\text { En bloc-LMX } \\
(n=267)\end{array}$ & $\begin{array}{l}\text { En bloc-LM1 } \\
(n=39)\end{array}$ & $\begin{array}{l}\text { Piecemeal-LMX (n } \\
=145)\end{array}$ & $\begin{array}{l}\text { Piecemeal-LM1 } \\
(n=4)\end{array}$ & $\begin{array}{l}\text { Total } \\
(n=455)\end{array}$ \\
\hline Median age (range), y & $66(30-88)$ & $64(37-84)$ & $68(40-84)$ & $53(51-64)$ & $66(30-88)$ \\
\hline Male/female patients, n & $147 / 120$ & $18 / 21$ & $94 / 51$ & $1 / 3$ & $260 / 195$ \\
\hline Median size (range), mm & $18(10-85)$ & $25(10-120)$ & $25(10-80)$ & $30(25-35)$ & $20(10-120)$ \\
\hline Location (right/left), n & $121 / 146$ & $27 / 12$ & $85 / 60$ & $1 / 3$ & $234 / 221$ \\
\hline \multicolumn{6}{|l|}{ Macroscopic type, $\mathrm{n}$} \\
\hline Polypoid (0-Is, Ip) & 139 & 19 & 77 & 3 & 238 \\
\hline Nonpolypoid (0-Ila, IIb, Ila + IIc) & 128 & 20 & 68 & 1 & 217 \\
\hline Method of resection (PO/EMR/ESD), $n$ & 29/138/100 & $1 / 15 / 23$ & $3 / 126 / 16$ & $0 / 3 / 1$ & $33 / 282 / 140$ \\
\hline Histologic type (LGA/HGA/TVA), n & $117 / 147 / 3$ & $11 / 27 / 1$ & $46 / 97 / 2$ & $3 / 1 / 0$ & $177 / 272 / 6$ \\
\hline Median follow-up (range), mo & $13.9(6.0-58.2)$ & $13.2(6.0-57.5)$ & $17.3(6.0-61.2)$ & $22.7(6.733 .4)$ & $14.8(6.0-61.2)$ \\
\hline
\end{tabular}

LMX, glands at lateral margin could not be evaluated; LM1, lateral margin positive for tumor cells; PO, polypectomy; EMR, endoscopic mucosal resection; ESD, endoscopic submucosal dissection; LGA, low grade adenoma; HGA, high grade adenoma; TVA, tubulovillous adenoma.

Table 2 Local recurrence data for each lateral margin status.

\begin{tabular}{|c|c|c|c|c|c|}
\hline & $\begin{array}{l}\text { En bloc-LMX } \\
(n=267)\end{array}$ & $\begin{array}{l}\text { En bloc-LM1 } \\
(\mathrm{n}=39)\end{array}$ & $\begin{array}{l}\text { Piecemeal-LMX } \\
(\mathrm{n}=145)\end{array}$ & $\begin{array}{l}\text { Piecemeal-LM1 } \\
(n=4)\end{array}$ & $\begin{array}{l}\text { Total } \\
(n=455)\end{array}$ \\
\hline Recurrent lesions, n (\%) & $6(2.2)$ & $2(5.1)$ & $22(15.2)$ & $0(0)$ & $30(6.6)$ \\
\hline Median time to recurrence (range), mo & $9.4(5.9-43.0)$ & $4.7(3.0-6.2)$ & $6.3(1.7-48.1)$ & & $6.3(1.7-48.1)$ \\
\hline Median size of recurrent lesion (range), $\mathrm{mm}$ & $6.5(3-20)$ & $5.5(5-6)$ & $5(2-10)$ & & $6(2-20)$ \\
\hline $\begin{array}{l}\text { Histologic type of recurrent lesion } \\
\text { (LGA/HGA/TVA/atypical glands) }\end{array}$ & $4 / 1 / 0 / 1$ & $1 / 1 / 0 / 0$ & $16 / 3 / 2 / 1$ & & $21 / 5 / 2 / 2$ \\
\hline
\end{tabular}

LMX, glands at lateral margin could not be evaluated; LM1, lateral margin positive for tumor cells; LGA, low grade adenoma; HGA, high grade adenoma; TVA, tubulovillous adenoma.

specimens were cut into slices 2 to $3 \mathrm{~mm}$ wide, like the ESD specimens. However, if the lesions were relatively small or had a narrow stalk, the specimens were cut into only two or three sections. Three specialists in gastrointestinal pathology evaluated the lesions. Usually, one of the three evaluated a lesion, and when discussion was needed, the other pathologists joined in. The lateral margin status was categorized according to the Japanese criteria [11] as follows: LM0, the lateral margin is free of tumor cells; LM1, the lateral margin is positive for tumor cells; and LMX, the glands at the lateral margin are damaged and cannot be evaluated for the presence of neoplasia. Cases in which the lateral margin had been damaged by piecemeal resection, and cases in which the sections were unsuitable for pathologic evaluation of the margins, were also categorized as LMX.

\section{Statistical analysis}

We used Fisher's exact test to compare categorical variables, and the independent $t$ test to compare continuous variables. The SPSS statistical software package, version 17.0 (SPSS Inc., Chicago, Illinois) was used for analysis, and a $P$ value of less than 0.05 was considered statistically significant.

\section{Results}

In the 389 lesions categorized as LM0, no evidence of local recurrence was noted. Of the 455 lesions categorized as LMX or LM1, 30 lesions showed local recurrence. The clinical characteristics of the 455 LMX and LM1 lesions are summarized in Table 1. For assessment, these lesions were divided into four groups according to their LM status (LMX or LM1) and type of endoscopic resection (en bloc or piecemeal). The median age of the patients with the 455 lesions categorized as LMX or LM1 was determined to be 66 years (range, $30-88$ ). The median tumor size was $20 \mathrm{~mm}$ (range, 10 - 120). En bloc resection was performed in 306 lesions (67.3\%). The median duration of follow-up after the initial endoscopic resection was 14.8 months (range, 6.0-61.2). In particular, we noted that the tumors of the piecemeal-LM1 group were larger and their follow-up was longer than those of the other groups.

The local recurrence rate for each lateral margin status appears in - Table 2. In total, 30 of the 455 lesions (6.6\%) showed local recurrence within the median period of 6.3 months (range, 1.748.1) from the initial endoscopic resection. In the en bloc resection group $(n=306)$, the local recurrence rate was lower among the patients with LMX lesions $(6 / 267,2.2 \%)$ than among the patients with LM1 lesions (2/39, 5.1\%); however, the difference between the local recurrence rates was not significant. In the piecemeal resection group $(n=149)$, the LMX lesions showed frequent recurrence $(22 / 145,15.2 \%)$. None of the LM1 lesions recurred $(0 / 4)$. The frequency of local recurrence of the piecemealLMX lesions was significantly higher than that of the en blocLMX lesions $(P<0.01)$. However, we did not identify any significant relationship between the clinicopathologic findings - such as size, histologic type, macroscopic type, and endoscopic resection method - and local recurrence in the en bloc-LMX group. The median time from the initial endoscopic resection to the development of recurrence was relatively longer in the en bloc-LMX group than in the other groups. The median size of the recurrent lesions in all the groups was $6 \mathrm{~mm}$. All 30 lesions that showed local recurrence were treated with an additional endoscopic resection. Histologic examination of the specimens indicated 23 cases of adenoma, 5 cases of intramucosal cancer, and 2 cases of atypical glands.

The clinical courses of the patients with recurrent lesions are summarized in Fig. 2 . The 6 patients in the en bloc-LMX group with recurrent lesions were treated with a second endoscopic re- 


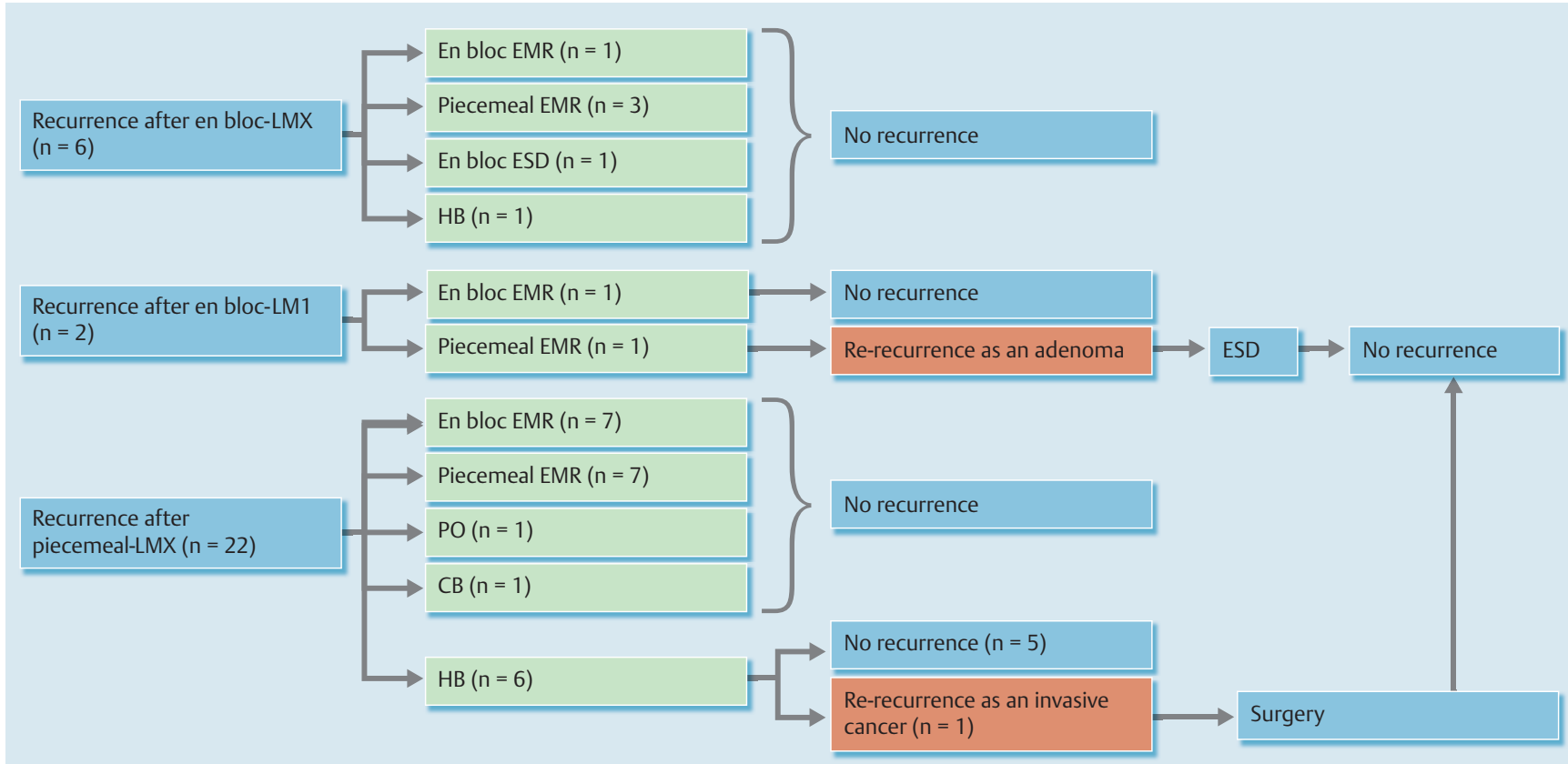

Fig. 2 Flowchart illustrating the clinical course of the recurrent lesions. EMR, endoscopic mucosal resection; LMX, glands at the lateral margin could not be evaluated; ESD, endoscopic submucosal dissection; HB, hot biopsy; LM1, lateral margin positive for tumor cells; PO, polypectomy; CB, cold biopsy..

section, and the lesions showed no further recurrence. Of the 2 patients with recurrent lesions in the en bloc-LM1 group, 1 had further recurrence after the second endoscopic resection and was then treated by ESD, following which no further recurrence was noted. Of the 22 patients with recurrent lesions in the piecemeal-LMX group, 1 had a lesion that showed further recurrence as an invasive cancer; this patient underwent surgical treatment and is currently doing well.

\section{Case}

The clinical course of one of the patients from the piecemeal-LMX group is described in Fig. 3. In this case, piecemeal EMR, involving more than 10 pieces, was used to remove a laterally spreading granular-type tumor (diameter, $50 \mathrm{~mm}$ ) in the cecum $(\bullet$ Fig. $\mathbf{3 a} \mathbf{a} \mathbf{b})$. On histologic examination, the lesion was found to be a well-differentiated intramucosal tubular adenocarcinoma with low grade atypia. No evidence of lymphovascular invasion was noted. Moreover, the vertical margins were found to be negative, and the lateral margin was categorized as LMX. After 3 months, a small, flat, elevated lesion was detected on the EMR scar, which was resected by hot biopsy ( $\bullet$ Fig. 3c). On a subsequent histologic examination, the lesion was determined to be a tubular adenoma. However, after 8 months, an irregular ulcerative lesion was detected at the same site and was diagnosed as a tubular adenocarcinoma, moderately differentiated type ( $\bullet$ Fig. $3 \mathrm{~d}$ ). The patient was treated with ileocecal resection. Histologic examination of the specimen showed that the cancer cells had extended to the serosa ( Fig. $3 \mathrm{e}$ ) but did not indicate any lymph node or distant metastasis. This patient has undergone regular follow-up.

\section{Discussion}

In the present study, we indicated a relationship between the lateral margin status of colorectal intramucosal neoplasms and the incidence of local recurrence after endoscopic resection. The main results of the present study are as follows: the local recurrence rate in the en bloc-LMX group (2.2\%) was significantly lower than that in the piecemeal-LMX group (15.2\%), and almost all the recurrent lesions were histologically proven to be intramucosal neoplasms and were treated successfully with an additional endoscopic resection.

In the clinical setting, we occasionally encounter intramucosal colorectal neoplasms that may have been endoscopically resected with an en bloc procedure, even though they are determined to be LMX on histologic examination. In such cases, if the endoscopists are confident of the absence of residual neoplastic tissue after resection, these lesions may be treated in the same manner as LMO lesions. However, this suggestion cannot be supported by evidence as only a few studies have examined the lateral margin status in such cases.

Previous studies have shown that the local recurrence rate after the en bloc resection of colorectal neoplasms is low [20-23]. However, these studies included not only intramucosal neoplasms but also submucosal invasive cancers. In addition, these reports focused only on the type of resection (en bloc or piecemeal) and did not focus on the resected margins in pathologic specimens. In contrast, in the present study, we included intramucosal neoplasms and also determined their lateral margin status. Thus, the information on the local recurrence rate provided in the present study may help clinical endoscopists to make decisions regarding treatment options.

The lateral margin of a resected specimen may be categorized as LMX for various reasons. First, electrocautery devices may damage the glands on the lateral margin of the lesion, and therefore, the pathologists may be unable to evaluate whether or not the glands are neoplastic. In such cases, there may be remnants of neoplastic tissue on the luminal side of the resection edge. However, the neoplastic tissue that remains is usually necrotic as a consequence of the thermal damage. This hypothesis is supported by several studies that have investigated the presence of residual tumor in surgically resected specimens from areas where 

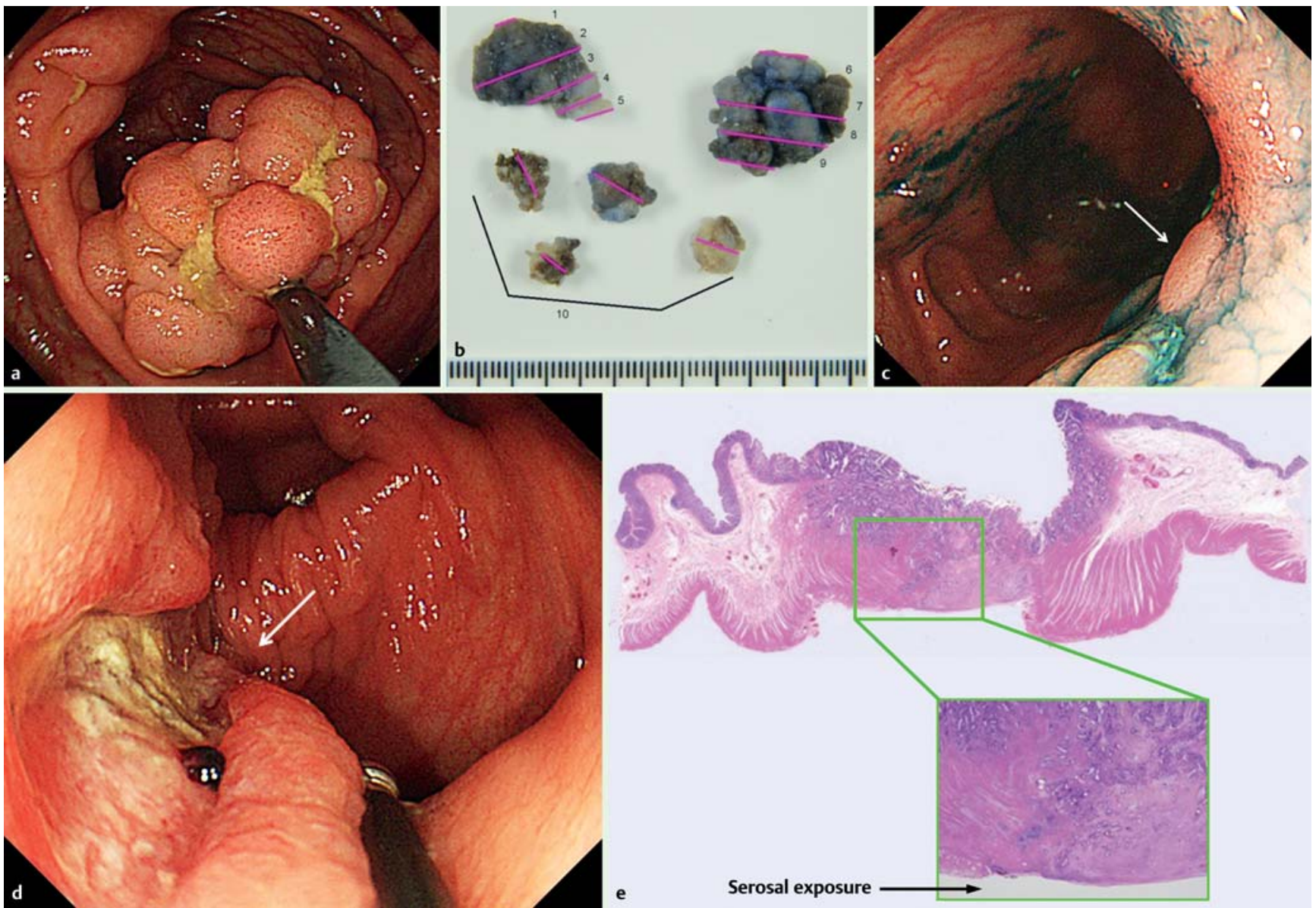

Fig. 3 Clinical course of a patient with a recurrent lesion. a A laterally spreading tumor, $50 \mathrm{~mm}$ in size, is detected in the cecum. b A piecemeal endoscopic mucosal resection (EMR) is performed to treat the lesion. c At 3 months after the piecemeal EMR, a recurrent lesion is detected at the site of the resection scar (arrow), which is then treated by hot biopsy. $\mathbf{d}$ After 8 months, further recurrence is noted (arrow). e lleocecal resection is performed to treat the recurrent lesion.

prior endoscopic resection had been performed. These studies indicated that superficial colorectal tumors that were endoscopically resected completely, but were histologically determined to have positive lateral margins, showed no residual cancer cells within the colorectal specimen after surgery [24,25].

Second, lesions may be categorized as LMX as a result of the histopathologic techniques used to evaluate the tissue, such as the manner in which the formalin-fixed specimen is cut. Endoscopically resected specimens are cut into several blocks that are 2 to $3 \mathrm{~mm}$ wide. In cases in which the resected specimen does not have sufficient non-neoplastic mucosa around the lesion, the initial and last en face slices contain only non-neoplastic tissue. Thus, pathologists can confidently determine if the lateral margin is tumor free. However, in cases in which the resected specimen is almost entirely composed of neoplastic tissue, the initial and last en face slices may also contain neoplastic tissue. Hence, the pathologists are unable to confidently determine whether the lesion has a negative lateral margin, and thus, the lesion is categorized as LMX [26]. However, because of the clear border that we observe between the colorectal lesion and normal mucosa, the potential for neoplastic tissue to remain within the lumen in such cases is believed to be low.

Third, lesions may be categorized as LMX as a result of other handling procedures. For instance, when the mucosa, muscularis mucosae, and submucosal layers are cut in the same manner, the pathologists can effectively evaluate the lateral margin. However, if the resection edge is not perpendicular to the mucosal-submucosal layer, the section exhibits an indeterminate layer structure. In such cases, the pathologist cannot effectively evaluate the lateral margin, and hence, the lesion is categorized as LMX. For an ESD specimen, the section is fixed on either a cork board or a rubber sheet with pins, then immersed in the fixative. This prevents the edges of the specimen from curling during fixation and enables better sectioning. However, with polypectomy and EMR specimens, the sections are usually immersed in a small bottle containing formalin and the lesion is not fixed on a board, which may make sectioning of the tissues difficult. We believe that these lesions with a disoriented layer structure may actually be LMO in most cases. Therefore, the possibility of the presence of tumor remnants is low in those cases in which the resection was en bloc but the specimens were pathologically classified as LMX. This is supported by the low recurrence rate in the en bloc-LMX group noted in the present study. Although we hypothesized that certain clinical factors, such as location, macroscopic type, and size of the initial lesion, may increase the difficulty of endoscopic resection and lead to higher local recurrence rates in the en blocLMX group, we could not identify any clinical risk factors that were related to local recurrence, which may have been because of the small sample size.

Previous studies have shown a high local recurrence rate in cases of piecemeal resection [19-23,27]. These studies included not only intramucosal neoplasms but also superficial submucosal in- 
vasive neoplasms. In the present case, we included only intramucosal neoplasms and excluded lesions that may have had other noncurative characteristics, such as a positive vertical margin and lymphovascular invasion.

The reasons for the high recurrence rate in piecemeal resection may be the following. First, the pathologist cannot reconstruct the specimen and so cannot evaluate the true lateral margin. Second, the thermal effect that is frequently noted in the specimens may hamper precise pathologic evaluation.

Certain Japanese guidelines have recommended that lesions with indeterminate lateral margins should be examined endoscopically within 6 to 12 months of the initial endoscopic resection [11]. In the present study, the local recurrence rate of lesions that were resected en bloc was low. Almost all the recurrent lesions were limited to the mucosal layer and were treated with an additional endoscopic resection. Hence, we believe that the lesions in the en bloc-LMX group can be treated similarly to LMO lesions when the endoscopist is confident about the completeness of the endoscopic resection.

However, we also recommend that patients undergoing piecemeal resections be followed carefully. In the present study, among the patients who underwent piecemeal resections, we observed a high local recurrence rate, and in one case, an invasive cancer was eventually diagnosed. The US Multi-Society Task Force on Colorectal Cancer and the American Cancer Society have recommended that endoscopic surveillance be performed within 3 to 6 months after the piecemeal resection of large colorectal polyps [28].

The present study has certain limitations. First, the study was retrospective in nature and was performed at a single institution. Second, the sample size was relatively small and the follow-up period was short. Third, only magnifying colonoscopy was used for the diagnosis of colorectal lesions and the detection of residual lesions. Several studies have indicated the efficacy of magnifying colonoscopy in predicting the completeness of endoscopic resection [29]. Thus, the local recurrence rates in the present study may differ from those in studies in which only conventional colonoscopy was used.

In conclusion, we noted that the local recurrence rate of lesions treated with en bloc resection and categorized as LMX was lower than that of lesions treated with piecemeal resection and categorized as LMX. Furthermore, almost all the recurrent lesions were successfully treated by an additional endoscopic resection. Thus, we believe that en bloc-LMX lesions can be treated similarly to en bloc-LMO lesions.

\section{Competing interests: None}

\section{References}

1 Deyhle P, Seuberth K, Jenny $S$ et al. Endoscopic polypectomy in the proximal colon. Endoscopy 1971; 3: 103-115

2 Deyhle P, Largiader F, Fumagalli S. A method for endoscopic electroresection of sessile colonic polyps. Endoscopy 1973; 5: 38-40

3 Kudo S. Endoscopic mucosal resection of flat and depressed type of early colorectal cancer. Endoscopy 1993; 25: 455-461

4 Ahmad NA, Kochman ML, Long WB et al. Efficacy, safety, and clinical outcomes of endoscopic mucosal resection: a study of 101 cases. Gastrointest Endosc 2002; 55: 390-396

5 Moss A, Bourke MJ, Williams SJ et al. Endoscopic mucosal resection outcomes and prediction of submucosal cancer from advanced colonic mucosal neoplasia. Gastroenterology 2011; 140: 1909-1918
6 Ohkuwa M, Hosokawa N, Boku $N$ et al. New endoscopic treatment for intramucosal gastric tumors using an insulated-tip diathermic knife. Endoscopy 2001; 33: 221 -226

7 Ono H, Kondo H, Gotoda T et al. Endoscopic mucosal resection for treatment of early gastric cancer. Gut 2001; 48: 225-229

8 Gotoda $\mathrm{T}$, Kondo $\mathrm{H}, \mathrm{Ono} \mathrm{H}$ et al. A new endoscopic resection procedure using an insulation-tipped electrosurgical knife for rectal flat lesions: report of two cases. Gastrointest Endosc 1999; 50: 560-563

9 Tamegai $Y$, Saito $Y$, Masaki $N$ et al. Endoscopic submucosal dissection: a safe technique for colorectal tumors. Endoscopy 2007; 39: 418-422

10 Saito Y, Uraoka T, Yamaguchi Yet al. A prospective, multicenter study of 1111 colorectal endoscopic submucosal dissections (with video). Gastrointest Endosc 2010; 72: 1217-1225

11 Japanese Society for Cancer of the Colon and Rectum. Japanese classification of colorectal carcinoma. 2nd edn. Tokyo, Japan: Lanehara; 2009

12 Watanabe T, Itabashi M, Shimada Yet al. Japanese Society for Cancer of the Colon and Rectum (JSCCR) guidelines 2010 for the treatment of colorectal cancer. Int J Clin Oncol 2012; 17: 1 - 29

13 Pohl E, Srivastava A, Bensen SP et al. Incomplete polyp resection during colonoscopy - results of the complete adenoma resection (CARE) study. Gastroenterology 2013; 144: 74-80

14 The Paris endoscopic classification of superficial neoplastic lesions: esophagus, stomach, and colon. Gastrointest Endosc 2003; 58: S3-S43

15 Kudo S, Hirota S, Nakajima T et al. Colorectal tumours and pit pattern. J Clin Pathol 1994; 47: 880-885

16 Fujii T, Hasegawa RT, Saitoh Y et al. Chromoscopy during colonoscopy. Endoscopy 2001; 33: 1036-1041

17 Matsuda T, Fujii T, Saito $Y$ et al. Efficacy of the invasive/non-invasive pattern by magnifying chromoendoscopy to estimate the depth of invasion of early colorectal neoplasms. Am J Gastroenterol 2008; 103 : $2700-2706$

18 Sano Y, Horimatsu T, Fu KI et al. Magnifying observation of microvascular architecture of colorectal lesions using a narrow band imaging system. Dig Endosc 2006; 18: 44-S51

19 Saito Y, Uraoka T, Matsuda T et al. Endoscopic treatment of large superficial colorectal tumors: a case series of 200 endoscopic submucosal dissections (with video). Gastrointest Endosc 2007; 66: 966-973

20 Iishi $H$, Tatsuta $M$, Iseki $K$. Endoscopic mucosal resection with submucosal saline injection of large sessile colorectal polyps. Gastrointest Endosc 2000; 51: 697-700

21 Higaki S, Hashimoto S, Harada K et al. Long-term follow-up of large flat colorectal tumors resected endoscopically. Endoscopy 2003; 35: $845-$ 849

22 Hotta K, Fujii T, Saito $Y$ et al. Local recurrence after endoscopic resection of colorectal tumors. Int J Colorectal Dis 2009; 24: 225-230

23 Saito Y, Fukuzawa M, Matsuda T et al. Clinical outcome of endoscopic submucosal dissection versus endoscopic mucosal resection of large colorectal tumors as determined by curative resection. Surg Endosc 2010; $24: 343-352$

$24 \mathrm{Kim} \mathrm{JH}$, Cheon JH, Kim TI et al. Effectiveness of radical surgery after incomplete endoscopic mucosal resection for early colorectal cancers: a clinical study investigating risk factors of residual cancer. Dig Dis Sci 2008; 53: $2941-2946$

25 Kim KM, Eo SJ, Shim SG et al. Risk factors for residual cancer and lymph node metastasis after noncurative endoscopic resection of early colorectal cancer. Dis Colon Rectum 2013; 56: $35-42$

26 Lauwers GY, Ban S, Mino M et al. Endoscopic mucosal resection for gastric epithelial neoplasms: a study of 39 cases with emphasis on the evaluation of specimens and recommendations for optimal pathologic analysis. Mod Pathol 2004; 17: $2-8$

27 Sakamoto T, Matsuda T, Otake Y et al. Predictive factors of local recurrence after endoscopic piecemeal mucosal resection. J Gastroenterol 2012; 47: 635-640

28 Winawer SJ, Zauber AG, Fletcher RH et al. Guidelines for colonoscopy surveillance after polypectomy: a consensus update by the US MultiSociety Task Force on Colorectal Cancer and the American Cancer Society. Gastroenterology 2006; 230: $1872-1885$

29 Hurlstone DP, Cross SS, Brown S et al. A prospective evaluation of highmagnification chromoscopic colonoscopy in predicting completeness of EMR. Gastrointest Endosc 2004; 59: 642-650 